\title{
A propos de la méthode Schmid - Bondzynski - Ratslaff
}

\author{
par \\ Robert GILLET \\ (Laboratoire Intercommunal de Bruxelles)
}

Dans une étude déjà ancienne $(*)$, M. J. Pien s'est appliqué à démontrer l'exactitude de la méthode Schmid-Bondzynski - Ratslaff (S-B-R-) recommandée par la Convention Internationale de Rome du 26 avril 1934, pour le dosage de la matière grasse dans les fromages. Pour ce faire, l'auteur a déterminé la teneur en matière grasse de nombreux fromages par la méthode S-B-R et la méthode au sable prise comme référence. Et il en conclut que « les deux méthodes donnent des résultats pratiquement identiques ".

Tout en nous ralliant à cette conclusion de bon sens, nous avons cru intéressant de revoir les chiffres à la lumière des méthodes statistiques, de manière à préciser la signification de la différence entre les moyennes observées. Le nombre élevé d'analyses permet en effet d'obtenir un bonne précision dans les estimations.

$$
\text { *** }
$$

Comme il s'agit de comparer deux méthodes de mesure en soumettant les mêmes individus à chacune de ces deux méthodes, nous pouvons considérer les deux échantillons statistiques comme associés par paires et traiter l'échantillon statistique constitué par les différences entre les deux individus de chaque paire. Le test d'égalité des deux moyennes sera en réalité un test de conformité à 0 de la moyenne des différences, et la différence entre les deux moyennes aura comme intervalle de confiance celui de la moyenne des différences.

\section{Première série d'essais : 20 fromages à pâte pressée}

Moyennes : méthode au sable : 15,482 Intervalle var. 12,22 - 18,77 méthode SBR : 15,507 » » $12,30-18,85$

(*) Le Lait, mai-juin 1948, p. 225. 
Différence entre les moyennes : 0,025

Variance estimée des différences : 0,0060

Test d'égalité des moyennes : $\frac{0,025}{v \frac{0,0060}{20}}=1,44$

L'écart entre les moyennes observées n'est pas significatif. Nous ne pouvons rejeter l'hypothèse d'égalité des moyennes réelles au niveau des populations infinies de mesures. Est-ce à dire que cette hypothèse est vérifiée ? Nullement car un test négatif ne constitue pas une preuve. Il est même infiniment probable qu'il existe une différence si petite soit-elle entre les résultats moyens réels des deux méthodes. L'évaluation de la puissance de notre test (qui dépend de la variance des différences et du nombre de mesures) montre d'ailleurs qu'au niveau de signification 0,05 (c'est-à-dire dans le cas où une différence est déclarée significative lorsqu'elle n'a pas plus que 5 chances sur 100 d'être dépassée par le seul effet du hasard de l'échantillonnage) on aurait encore 1 chance sur 10 de ne pas mettre en évidence (d'obtenir un test négatif pour) une différence réelle de 0,060 .

\section{Intervalle de confiance de la différence}

Mais dans la pratique, il importe surtout d'évaluer cette différence réelle. La meilleure estimation en sera la différence des moyennes observées : 0,025 que nous entourerons d'un intervalle de confiance à 95 p. 100. Les limites de confiance seront (pour un degré de confiance de 95 p. 100) :

$$
0,025 \pm 2,09 \vee \frac{0,0060}{20}=-0,011 \text { et } 0,061
$$

Cet intervalle de confiance a 95 chances sur 100 de couvrir la vraie valeur (sur un grand nombre d'intervalles calculés de la même façon sur le même nombre d'échantillons statistiques, 95 p. 100 d'entre eux couvriraient la vraie valeur). La présence de la valeur 0 à l'intérieur de cet intervalle confirme l'acceptation de l'hypothèse d'égalité des moyennes réelles.

C'est sur cet intervalle que le chimiste doit se baser pour décider si les deux méthodes donnent des résultats équivalents, compte tenu de la précision de la mesure.

\section{Deuxième série d'essais : $\mathbf{4 0}$ fromages moisis}

Moyennes : méthode au sable : 13,694 Intervalle var. 11,18 - 20,02 méthode SBR : 13,993 " " $11,20-20,05$

Différence entre les moyennes : 0,029 
Variance estimée des différences : 0,0031

Test d'égalité des moyennes : $\frac{0,029}{v \frac{0,0031}{40}}=3,22$

L'écart entre les moyennes observées est significatif : il aurait moins d'une chance sur 100 d'être dépassé par le seul effet du hasard de l'échantillonnage. Nous pouvons donc rejeter l'hypothèse d'égalité des moyennes réelles au niveau des populations infinies de mesures. Faut-il nous en inquiéter ? En aucune façon, une différence entre les moyennes réelles est dans l'ordre des choses, nous l'avons vu ; et si, à l'encontre du cas précédent, nous avons pu la mettre en évidence, c'est grâce à une variance plus réduite et surtout à un nombre plus élevé d'analyses : le test avait cette fois 9 chances sur 10 de déceler une différence réelle égale à 0,030 .

Intervalle de confiance de la différence

A nouveau, il nous importe bien davantage d'estimer la différence en question. Les limites de confiance en seront, pour un degré de confiance de 95 p. 100 :

$$
0,029 \pm 2,02 \vee \frac{0,0031}{40}=0,011 \text { et } 0,047
$$

Et nous aboutissons à cette constatation, sans doute paradoxale que, tout en ne couvrant pas la valeur 0 (ce qui confirme le rejet de l'hypothèse d'égalité), l'intervalle de confiance est plus favorable que précédemment à la thèse de l'équivalence des méthodes car c'est en fait la limite de confiance la plus éloignée de 0 qui nous sert de critère d'équivalence, compte tenu de la précision de la mesure.

\section{Interprétation}

S'il n'y a pas d'interaction, c'est-à-dire si la différence entre les moyennes réelles ne diffère pas d'un fromage à l'autre, nous pouvons estimer la précision des mesures individuelles car la variance de ces mesures vaudra la moitié de la variance des différences. Et nous pouvons dès lors calculer un intervalle de confiance pour la mesure. Le chimiste dispose donc de tous les éléments nécessaires à l'énoncé d'un jugement :

- limite supérieure pour la différence réelle : 0,05,

- variance estimée des mesures $\frac{0,0031}{2}=0,0015$,

et limites de confiance

pour une mesure $x: x \pm 2,02$. $\vee 0,0015=x \pm 0,08$. 
Le problème se pose donc de la façon suivante : étant donné que toute mesure est entachée d'une imprécision telle qu'elle doive s'entourer d'un intervalle de confiance de 0,16 de largeur, convient-il de se préoccuper d'une erreur systématique en excès pouvant au pis atteindre 0,05 ? Nous pensons pour notre part que non, mais il est toujours loisible au chimiste de soustraire 0,03 de chacune de ses mesures.

\section{S u m m a ry}

Comparison of the results of the Schimd-Bondzinsky-Ratslaff method with those of the "sand-method " for determination of fat in cheese.

The A. treats statistically the analytical numbers published, years ago, by $\mathrm{Mr} \mathrm{J}$. Pien and demonstrates that the two methods give slightly different mean results.

Nevertheless, the difference found is negligible in consideration of the precision of the analytical methods.

Reçu pour publication en décembre 1968. 